\title{
Development of Sensitization in the Escape Locomotion System in Aplysia
}

\author{
Mark Stopfer and Thomas J. Carew \\ Departments of Psychology and Biology, Yale University, New Haven, Connecticut 06520
}

The development of several forms of nonassociative learning (habituation, dishabituation, and sensitization) has previously been examined in the gill and siphon withdrawal reflex of Aplysia. In the present study we analyzed the development of one of these forms of learning, sensitization, in a different response system in Aplysia, escape locomotion. A broad range of juvenile stages was examined: stages 10,11 , early 12 , late 12 , and 13 (early adult). We found that sensitization was completely absent in early developmental stages, not appearing until late stage 12. This stage of development is particularly interesting because it is at this same point that (1) sensitization first appears in the gill and siphon withdrawal reflex (Rankin and Carew, 1987), and (2) the cellular analog of sensitization first emerges in the CNS (the abdominal ganglion) of juvenile Aplysia (Nolen and Carew, 1987).

The fact that sensitization emerges synchronously in the escape locomotion system and the gill withdrawal system is striking because the 2 response systems differ markedly in their intrinsic developmental timetables, response topography, and underlying neural circuitry. Thus, the emergence of sensitization in both systems at the same late stage of juvenile development suggests the possibility that a single, unified process during development may be responsible for the simultaneous expression of sensitization.

The development of learning and memory has been analyzed in considerable detail in defensive reflexes of Aplysia (Peretz and Lukowiak, 1975; Rayport and Camardo, 1984; Nolen and Carew, 1987, 1988; Nolen et al., 1987; Rankin and Carew, 1987a, 1988; Rankin et al., 1987). The gill and siphon withdrawal reflex in particular has been examined both because it exhibits a variety of forms of learning and memory (Pinsker et al., 1970, 1973; Carew, et al., 1981a, 1983; Hawkins, et al., 1985) and because its neural circuitry is very well understood (Kupfermann and Kandel, 1969; Kupfermann et al., 1974; Byrne, et al., 1978; Hawkins et al., 1981). However, in addition to the gill and siphon withdrawal reflex, Aplysia possesses a number of other response systems that are also useful for a develop-

\footnotetext{
Received Mar. 3, 1987; revised June 15, 1987; accepted June 17, 1987.

We wish to thank Deda Gillespie for her excellent help with data collection and our colleagues Emilie Marcus, Thomas Nolen, Catharine Rankin, and William Wright for their helpful criticism of an earlier version of the manuscript. We also are very grateful to the Howard Hughes Medical Institute, and to Tom Capo, for generously supplying juvenile Aplysia. This work was supported by NIH BRSG Grant 507-RR-075015 and by NSF Grant BNS 8311300 (to T.J.C.).

Correspondence should be addressed to Thomas J. Carew, Department of Psychology, Yale University, Box 11 A Yale Station, New Haven, CT 06520.

Copyright (C) 1988 Society for Neuroscience $0270-6474 / 88 / 010223-08 \$ 02.00 / 0$
}

mental analysis. One such behavior is escape locomotion, a dramatic stereotypic response in which an animal rapidly moves away from a source of noxious stimulation. Escape locomotion offers a variety of advantages for a developmental analysis: (1) It is expressed very early in Aplysia development (as early as stage 8 , unpublished observations); (2) it is simple to quantify in a completely unrestrained animal (Hening et al., 1979; Walters et al., 1979); (3) the response in the adult is known to be modulated by several different forms of both nonassociative and associative learning (Walters et al., 1978, 1979, 1981; Carew et al., 1981 b); and (4) the neural circuitry underlying escape locomotion in the adult has been examined in some detail (Jahan-Parwar and Fredman, 1978a, b; Hening et al., 1979). Thus, escape locomotion provides an appropriate behavioral system in Aplysia in which a developmental analysis could provide important insights into the general question of the ontogenetic assembly of different forms of learning.

One type of learning exhibited in a number of different behaviors in Aplysia is sensitization, a simple form of learning in which a strong stimulus facilitates responding to subsequent stimuli. Sensitization in the gill and siphon withdrawal reflex emerges surprisingly late in juvenile development, several weeks after the emergence of habituation and dishabituation (Rankin and Carew, 1986, 1988). Sensitization is also known to be exhibited in the escape locomotion response in the adult (Walters et al., 1978). Thus, this raised the interesting question of whether sensitization of escape locomotion emerges at the same late stage of juvenile development as in the gill and siphon withdrawal reflex or whether it emerges according to a different developmental timetable.

A comparison between the gill and siphon withdrawal reflex and escape locomotion is interesting for 2 reasons. First, the behaviors have different birthdates. Aplysia exhibit escape locomotion soon after metamorphosis; it has been observed in animals as early as stage 8 . In contrast, siphon and gill withdrawal emerge several days later in stages 9 and 10, respectively (Kriegstein, 1977a; Rankin et al., 1987). Second, the response topography and underlying neural circuitry for the 2 behaviors are quite different. The gill and siphon withdrawal reflex is a graded response whose magnitude and time course are directly related to the magnitude and duration of the eliciting stimulus (Carew and Kandel, 1977; Rankin et al., 1987). In contrast, escape locomotion is a centrally programmed, fixed action pattern mediated by an oscillatory neural circuit (Kandel, 1976; Hening et al., 1979). Thus, gill and siphon withdrawal and escape locomotion differ both in their developmental timetables and in their behavioral and cellular complexity.

Despite these marked differences, we have found that sensi- 
A
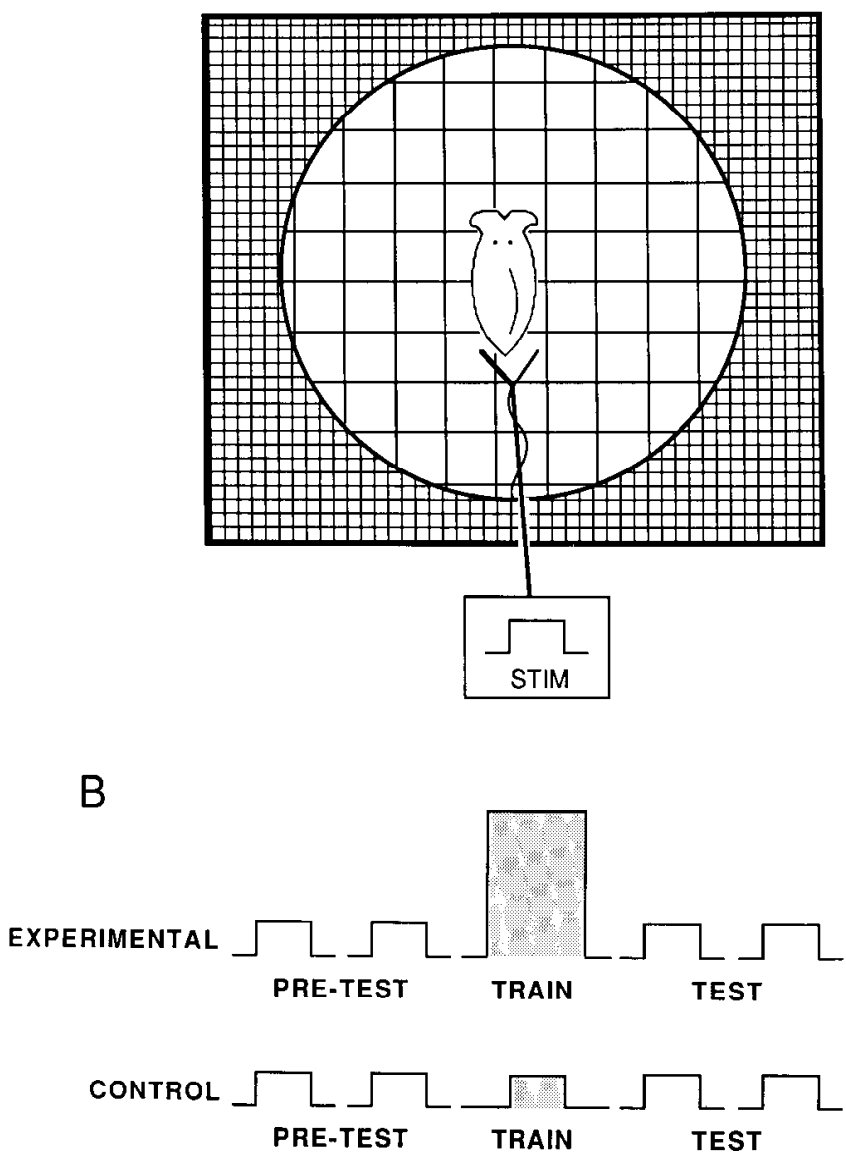

Figure 1. Schematic illustration of experimental procedure. A, Test arena. Different-sized open-field test arenas were appropriately scaled for each developmental stage. Juvenile stages 10 to early 12 were observed through a boom-mounted dissecting microscope that could be kept centered over the animal (the magnified center circle illustrates the animal as viewed through the microscope). Stimuli were delivered to the tail between spanning electrodes that did not contact the tail. Escape locomotion was quantified by the number of boxes entered during an observation period (see Materials and Methods for details).

$B$, Experimental procedure. Both experimental and control groups first received 2 identical mild PRE-TEST stimuli, separated by $2 \mathrm{~min}$. After each stimulus the distance traveled by each animal was recorded. Next, a second experimenter delivered 1 of 2 training stimuli: the $e x$ perimental groups received a strong sensitizing stimulus ( 5 times threshold for 1 sec; shaded bar); the Control group received another mild stimulus, identical to pretest (shaded bar). Finally, the first experimenter delivered 2 more mild (TEST) stimuli, identical to pretest. Each animal contributed a single score, the mean test response expressed as a percentage of the mean pretest response.

tization emerges in the escape locomotion system at the same late stage of juvenile development as in the gill and siphon withdrawal reflex. This suggests the possibility that sensitization emerges as a single, unified process during development and is expressed in a variety of response systems simultaneously. Some of the results described in this paper have previously been presented in preliminary form (Stopfer and Carew, 1986).

\section{Materials and Methods}

Animals. One hundred and ten Aplysia californica were used in the experiments. All animals were laboratory-cultured specimens obtained from the Howard Hughes Medical Institute, Woods Hole, MA. Juvenile animals (stages 10-12, see below) were maintained in groups on seaweed
(Gracilaria) in $50 \mathrm{ml}$ plastic centrifuge tubes filled with seawater $\left(15^{\circ} \mathrm{C}\right.$ ) obtained from Woods Hole. Adult Aplysia (stage 13) were maintained at room temperature $\left(20-22^{\circ} \mathrm{C}\right)$ in groups of 4 in perforated pans floating in a 300 gal tank of artificial seawater (Instant Ocean). All animals were housed at least $24 \mathrm{hr}$ prior to an experiment.

Juvenile Aplysia can be divided into distinct stages based on external morphological criteria (Kriegstein, 1977a; Rankin and Carew, 1986, 1987a, 1988). In the present study we examined 5 developmental stages: 10,11 , early 12 , late 12 , and early 13 (young adults). Stage 10 animals are $1.0-2.0 \mathrm{~mm}$ in length, are largely enclosed in an externally visible shell, and lack rhinophores. Stage 11 animals are $2.0-4.0 \mathrm{~mm}$ in length and have small rhinophore buds and parapodia that are almost large enough to meet atop the shell. Early stage 12 animals are $7.0-10.0 \mathrm{~mm}$ in length, have a shallow genital groove, distinct rhinophores, and overlapping parapodia; they are also broader than the slender stage 11 animals. Late stage 12 animals are $20.0-30 \mathrm{~mm}$ in length, have a distinct genital groove, and are generally much larger than the early stage 12 animals. The transition from stage 12 to 13 (adult) is difficult to identify precisely, as it is defined behaviorally (as the onset of sexual maturity). In this study, early stage 13 (young adult) animals were defined as 60.0 $110.0 \mathrm{~mm}$ in length. (For details of staging criteria see Kriegstein, 1977a; Rankin and Carew, 1986, 1987a, 1988).

Behavioral procedures. Each trial in the experiment began by manually centering the animal within the open field chamber (Fig. 1A). Animals were always rested for $90 \mathrm{sec}$ after centering before test or training stimuli were delivered. The experimental procedure used to assess sensitization is shown in Figure $1 B$. There were 3 phases to the experiment: (1) pretesting, (2) training, and (3) testing. In the pretest phasc, stationary animals first received 2 weak pretest stimuli (mild tail shock, see below), each followed by a 2 min observation period during which the distance traveled by the animal was recorded at $10 \mathrm{sec}$ intervals. Distance traveled was quantified as the number of boxes entered during each $10 \mathrm{sec}$ epoch. Following the pretest, for the training phase, a second experimenter randomly assigned the animal to either the Sensitization group or the Control group. The Sensitization group received sensitization training (strong electric shocks to the tail, see below), while the Control group received another weak test stimulus. The training stimulus was delivered by the second experimenter, who was not aware of the animal's pretraining scores. Following training, in the test phase, both groups received another 2 test stimuli, identical to pretest, delivered by the first experimenter, who was not aware of the group assignment of the animal. Thus, there were 2 blind proccdurcs: (1) The testing experimenter was unaware of the experimental condition of the animals; and (2) the training experimenter was unaware of the pretest scores of the animals, and it was the training experimenter who randomly generated the group assignments.

Both testing and training stimuli consisted of a train of electric pulses applied via spanning electrodes to the tail (see Fig. $1 A$ ). Stimulus strengths were normalized to each animal by determining the animal's threshold response for tail withdrawal (to a $0.5 \mathrm{sec}$ stimulus) $5 \mathrm{~min}$ before each experiment. In most experiments, 2 stimulus intensities were used: weak test stimuli $(0.5 \mathrm{sec}$ duration) were 1.5 times threshold intensity (stimuli ranging from 4 to $25 \mathrm{~V}$, depending upon the size of the animal); and strong sensitizing stimuli ( $1.0 \mathrm{sec}$ duration) were 5 times threshold intensity (range, 12-80 V). Stimulus intensities were determined by pilot studies that established the weakest intensity of test stimuli capablc of reliably eliciting locomotion, and the strongest intensity of sensitizing stimuli that could be delivered without causing physical damage to the tail. Animals at all stages responded to the strong sensitizing stimulus (but not the weak test stimulus) with a burst of ink and vigorous escape locomotion. Occasionally animals did not reliably move in response to the weak, pretest stimuli. These animals were excluded from the study before their group identities were determined. A total of 4 animals $(4 \%$ of all animals studied) were excluded by this criterion. In an additional experiment, a range of sensitizing stimulus intensities ( 3 and 5 times tail withdrawal threshold) were used to explore the presence of sensitization in stage 11 animals.

Animals at different developmental stages typically moved at different rates: in response to the mild test stimulus, animals traveled at rates of $1.0-20 \mathrm{~cm} / \mathrm{sec}$. For this reason, appropriately scaled open-ficld test chambers were prepared for each stage. Stage 10 Aplysia were tested in a glass petri dish $(14 \mathrm{~cm}$ diameter $\times 1 \mathrm{~cm}$ deep $)$ fitted with a thin Lucite floor. Stage 11 and early stage 12 Aplysia were tested in a Lucite tank $(42 \times 42 \times 1 \mathrm{~cm})$. Both the petri dish and the Lucite tank were placed on graph paper with millimeter divisions. Animals in stages 10 to early 


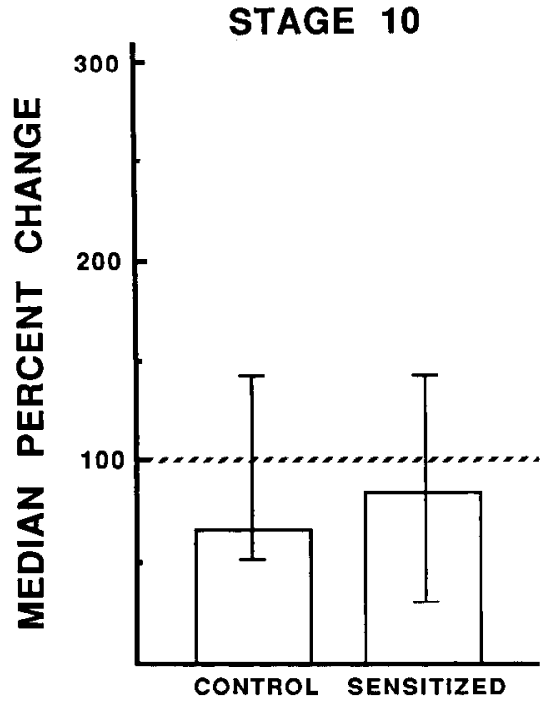

Figure 2. Stage 10 animals. No sensitization is evident in experimental (sensitized) animals cither by comparison with their own pretest scores or by comparison with control test scores. In this and subsequent figures, data are represented as median percentage change scores \pm interquartile range. The dotted line indicates the (normalized) pretest level ( $n=10$ per group).

12 were observed through a dissecting microscope mounted on a freely moving arm that permitted constant tracking of the animals. Constantlevel illumination of the test area was achieved by fiber-optic light guides attached to (and moving with) the microscope head. Late stage 12 animals were directly observed in a styrofoam tank $(40 \times 32 \times 4 \mathrm{~cm})$ fitted with a centimeter-division, grid-covered Lucite floor. Adult Aplysia were capable of relatively rapid locomotion and were therefore tested in a large, inflatable open-field chamber (a "kiddie pool" with an aquatic Disney motif) containing a grid-covered $(\mathrm{cm})$ Lucite floor. These larger animals were observed directly.

Scoring. On each trial, distance scores were defined as the total distance traveled during each of the 2 observation periods. Some adult animals occasionally reached the wall of the chamber before the conclusion of the 2 min observation period. In these instances, their scores were computed as the total distance traveled before the animals reached the side of the chamber. Each animal contributed a single score: The 2 pretest distance scores were averaged, as were the 2 test scores. The mean test score was then expressed as a percentage of the mean pretest score.

Statistical procedures. As the data were non-normally distributed, nonparametric statistics were used for the analyses. Between-group comparisons were made by means of Mann-Whitney $U$ tests, and for a summary analysis, a $\chi^{2}$ test was used; within-group comparisons were made by means of Wilcoxon signed-rank tests (Siegel, 1956). Extensive pilot studies permitted predicting the direction of experimental effects; therefore, all probability values are one-tailed.

\section{Results}

The primary focus of this study was to examine the time course of emergence of sensitization in the escape locomotion system of Aplysia. Five developmental stages were examined: stages 10, 11 , early 12 , late 12 , and early stage 13 .

\section{Stage 10}

The results from stage 10 animals are shown in Figure 2. In this and subsequent stages, within-group comparisons were made by assessing the median percentage change of the average test scores relative to the average pretest scores (see Materials and Methods). Animals at this stage showed no evidence of sensitization. A within-group comparison showed that neither the Sensitization group nor the Control group exhibited an increase

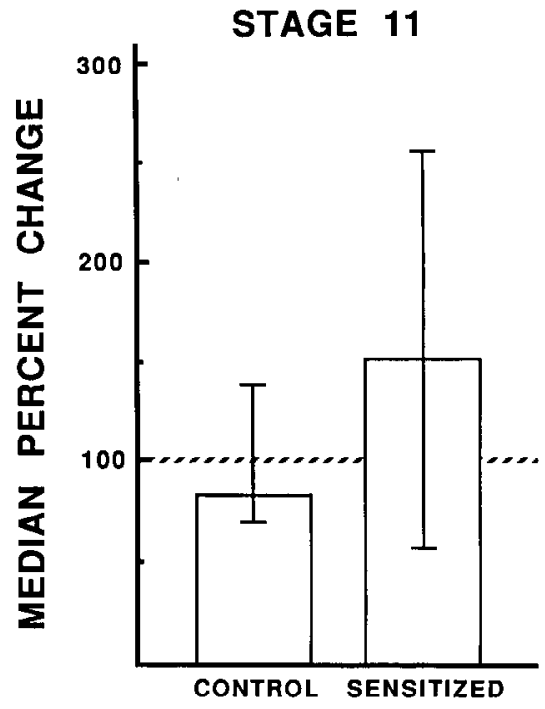

Figure 3. Stage 11 animals. As in stage 10 animals, stage 11 experimental animals show no significant sensitization either in comparison to their own pretest scores or in comparison to control test scores $(n=$ 10 per group).

in escape locomotion following training. The Sensitization group $(n=10)$ exhibited a median percentage change of $80.5(T=$ 21.5 , NS), while the Control group $(n=10)$ showed a median percentage change of $64.0(T=19.5$, NS). Moreover, a betweengroup comparison showed that there was no significant difference between Sensitization and Control groups ( $U=45$, NS). Finally, of those animals receiving sensitization training, only $40 \%$ showed an increase over their pretest scores, while $30 \%$ of control animals showed an increase. Thus, these results indicate that sensitization of escape locomotion was lacking in stage 10 animals.

\section{Stage 11}

The results from stage 11 animals are shown in Figure 3. Animals at this stage also showed no sensitization. A within-group comparison showed that neither the Sensitization group nor the Control group exhibited a significant increase in escape locomotion following training. There was considerable variability in the Sensitization group $(n=10)$, which exhibited a median percentage change of 157.0. However, this change was not significant $(T=20$, NS). The Control group $(n=10)$ showed a median percentage change of 85.5 ( $T=21$, NS). In addition, there was no significant difference between Sensitization and Control groups ( $U=36$, NS). Finally, of those animals receiving sensitization training, $60 \%$ showed an increase over their pretest scores, while $40 \%$ of control animals showed an increase. Thus, stage 11 animals also lack sensitization.

In experiments in which sensitization is found to be absent, it is important that our use of a "maximum" sensitizing stimulus intensity gives every chance for sensitization to be expressed. It is possible that the strong tail shock we used (5 times tail withdrawal threshold, see Materials and Methods) might recruit an inhibitory process (Rankin and Carew, 1987b, 1988; Nolen and Carew, 1987) that could mask sensitization and that a more moderate intensity shock might, in fact, be a more effective sensitizing stimulus. To examine this question, we carried out a second experiment in stage 11 animals in which 2 different stimulus intensities ( 3 and 5 times tail withdrawal threshold) 


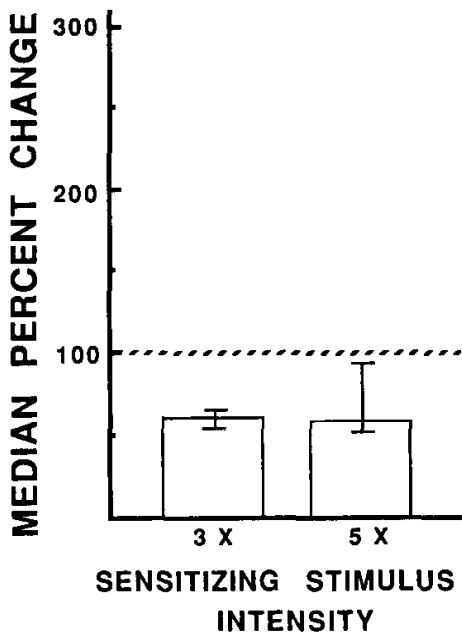

Figure 4. Effect of varying sensitizing stimulus intensity. Separate groups of stage 11 animals ( $n=5$ per group) received a sensitizing stimulus of either 3 or 5 times tail withdrawal threshold. Neither group showed response facilitation.

were used in different groups of animals $(n=5$ each). The results are shown in Figure 4. Neither group exhibited sensitization: the posttest scores of the $5 \times$ group were reduced following tail shock to a median percentage change of $63 \%$ of pretest, and the $3 \times$ group median posttest scores were $69 \%$ of pretest. Data from the previous experiment (Fig. 3) had shown that even weaker stimuli are also incapable of producing sensitization, since the control group, which received a training stimulus of 1.5 times tail withdrawal threshold, also showed a reduction in responding following tail shock. Thus, we have explored a range of sensitizing intensities ( $1.5,3$, and 5 times threshold) and found sensitization absent. Taken collectively, these data show that sensitization in stage 11 animals is not contingent upon stimulus intensity; stage 11 animals are not capable of sensitization.

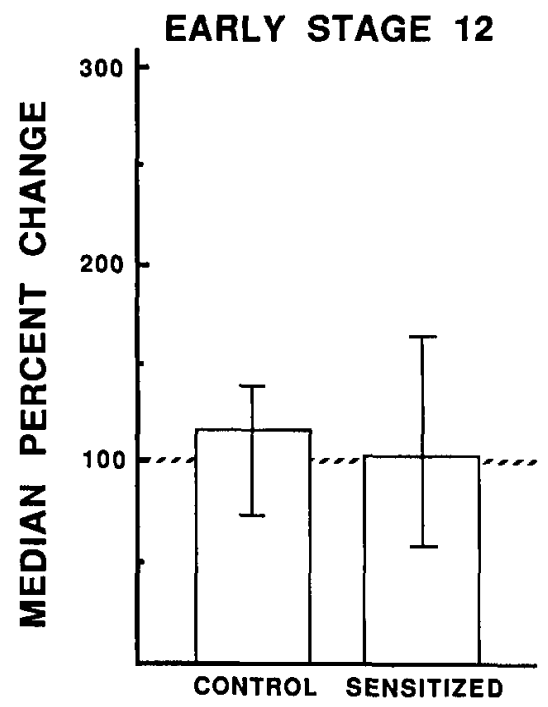

Figure 5. Early stage 12 animals. As in earlier stages, no sensitization is evident in experimental animals, either by comparison with their own pretest scores or by comparison with control test scores $(n=10$ per group).

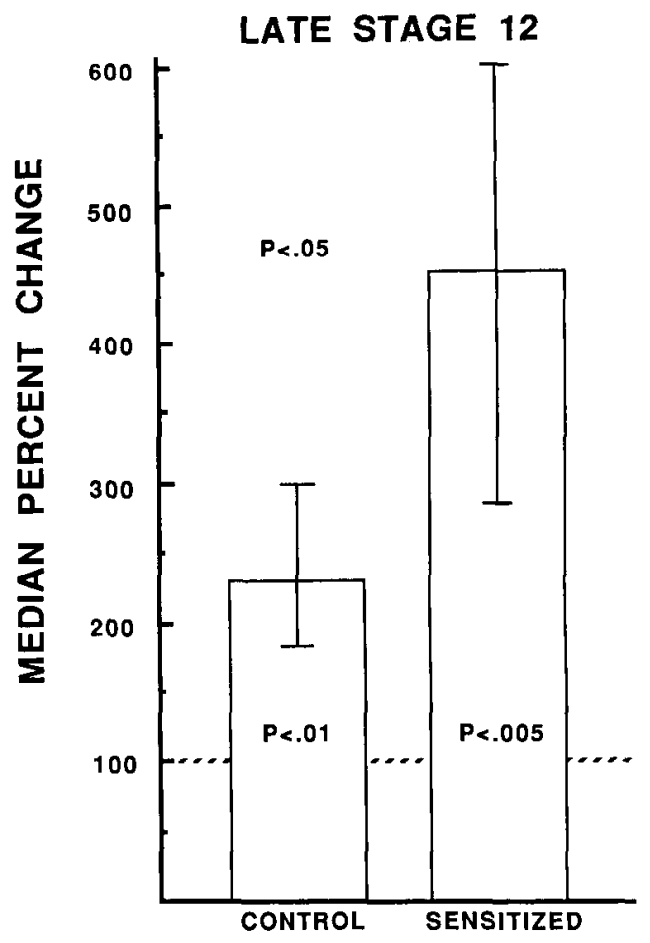

Figure 6. Late stage 12 animals. Both control and sensitized groups show significant increases over pretest scores (controls: $p<0.01$; sensitized: $p<0.005$; see text for discussion). However, experimental animals $(n=10)$ exhibit significantly greater sensitization than control animals $(n=10 ; p<0.05$ ), indicating that sensitization training produced significant response facilitation in this stage. (Note expanded ordinate compared to other figures.)

\section{Early stage 12}

The results from early stage 12 animals are shown in Figure 5 . As in previous stages, there was no sensitization exhibited in this developmental stage. A within-group comparison revealed that neither group exhibited a significant increase in escape locomotion after training. The Sensitization group $(n-10)$ had a median percentage change of $102.0(T=24, \mathrm{NS})$, and the Control group $(n=10)$ a median percentage change of $116(T=$ 19.5 , NS). Moreover, there was no significant difference between Sensitization and Control groups ( $U=46$, NS). Finally, of those animals receiving sensitization training, $50 \%$ showed an increase over their pretest scores, while $60 \%$ of control animals showed an increase.

\section{Late stage 12}

The results from late stage 12 animals are shown in Figure 6. In contrast to previous stages, sensitization was present at this developmental stage. A within-group comparison revealed that the Sensitization group $(n=10)$ exhibited a significant increase in escape locomotion following training, to a median percentage change of $450.0(T=0, p<0.005)$. Interestingly, the Control group $(n=10)$ also showed a significant increase, to a median percentage change of $233.0(T=5, p<0.01)$. These data may indicate that the handling procedures and repeated test stimulation at this developmental stage were sufficient to induce some sensitization in the Control group. However, a between-group comparison revealed that the Sensitization group exhibited a significantly greater increase in locomotion in the test period than the Control group $(U=26, p<0.05)$. Thus, although 


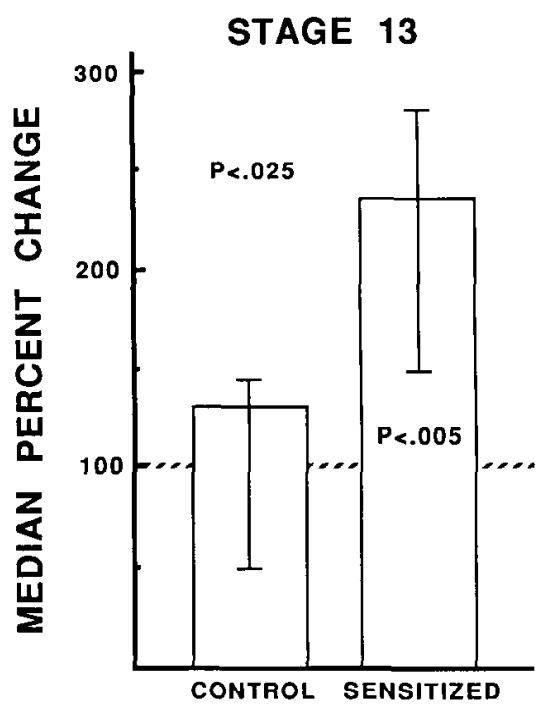

Figure 7. Early stage 13 animals. Experimental animals $(n=9)$ exhibit significant sensitization; both in comparison with their own pretest scores $(p<0.005)$ and in comparison to control test scores $(p<0.025)$. Control animals $(n=10)$ show no significant changes.

factors such as handling and test stimulation may contribute to the sensitization observed in the Sensitization group, they cannot completely account for the appearance of sensitization at this stage, since the strong stimulus during sensitization training induced significantly greater response enhancement than did handling and test stimulation. Finally, of the group receiving sensitization training, $100 \%$ showed an increase over prescores, while of the Control group, $80 \%$ showed an increase.

\section{Early stage 13}

The results from early adult, stage 13 animals are shown in Figure 7. As in the previous stage (late 12), and in contrast to the earlier stages, sensitization was evident in early adult animals. A within-group comparison revealed that the Sensitization group $(n=9)$ exhibited a significant increase in locomotion following training, to a median percentage change of $232.0(T=$ $0, p<0.005)$. Control animals $(n=10)$ did not exhibit a significant increase; they showed a median percentage change of $135.0(T=21, \mathrm{NS})$. Moreover, the Sensitization group showed significantly greater test responding than the Control group $(U=$ $17, p<0.025)$. Finally, of those animals receiving sensitization training, $100 \%$ showed an increase over pretest scores, while $70 \%$ of control animals showed an increase.

A summary of the results from all developmental stages is presented in Figure 8, which shows the data from animals that received sensitization training at each stage. The results are expressed in terms of the percentage of animals that exhibited increased responding following training, compared to their pretest responding. If sensitization is absent at a particular stage, one would expect that approximately $50 \%$ of the animals in that stage would show an increase in responding during the test (compared to the pretest) on the basis of chance alone (dashed line in Fig. 7). In examining these percentages across the different juvenile stages, a clear developmental trend emerges: in stages 10,11 , and early 12 , there is little deviation from the expected $50 \%$ level $(40,60$, and $50 \%$, respectively). However, both in stages late 12 and early $13,100 \%$ of animals receiving sensitization training showed increased responding in the test period.

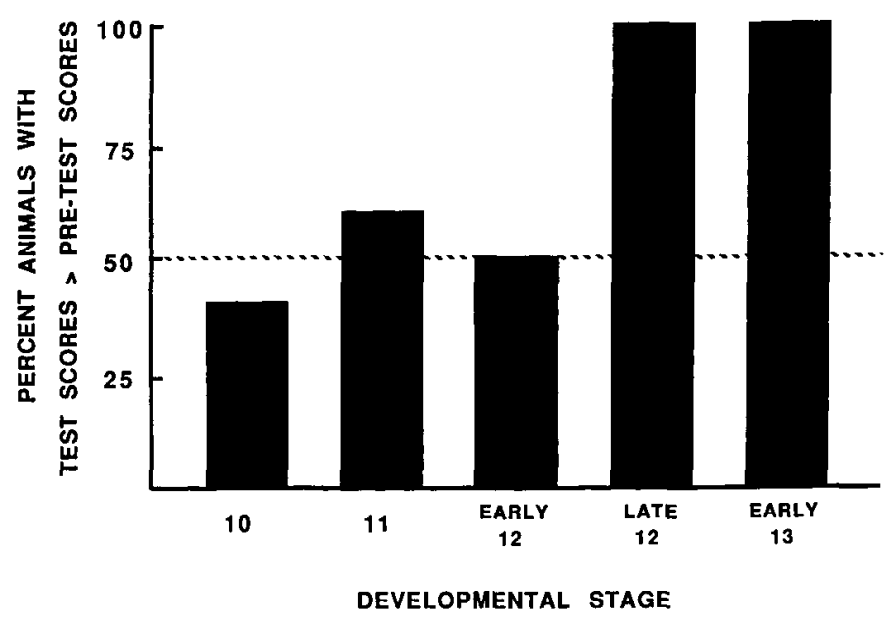

Figure 8. Summary of all developmental stages. Data are expressed as the pcrcentage of cxpcrimcntal animals within each stage exhibiting test scores greater than their pretest scores. The dotted line through the $50 \%$ level represents the percentage of animals within each group that would be expected to show an increase in responding (compared to pretest level) on the basis of chance alone. A significant developmental trend is exhibited $(p<0.01)$, indicating that sensitization of escape locomotion emerges in late stage 12 of juvenile development.

A statistical analysis of the distribution of these percentages across developmental stages revealed a significant increase in the later juvenile stages $\left[\chi^{2}(4)=106, p<0.01\right]$. Thus, taken collectively, our results show that sensitization of escape locomotion in Aplysia emerges in late stage 12 of juvenile development.

\section{Discussion}

\section{Development of sensitization}

In most animals, discrete forms of learning are expressed in a variety of different response systems. Thus, in the same animal, habituation can be independently observed in responses ranging from flexion reflexes to orienting responses, and classical conditioning can be independently observed in responses ranging from salivation to eye blink (Mackintosh, 1974). An important issue, then, in the development of learning is whether there is a single, unitary developmental sequence for the emergence of a particular form of learning or whether there are multiple sequences, such that each response system has its own independent development timetable for that form of learning. Sensitization is a simple form of learning expressed in several different response systems of Aplysia. Thus an analysis of the development of sensitization in different response systems in Aplysia could provide important insights into the general question of whether learning develops as a unitary process.

The developmental timetable for sensitization in the gill and siphon withdrawal reflex in Aplysia is already known. Sensitization in this reflex emerges quite late in juvenile development, in late stage 12 (Rankin and Carew, 1988). Moreover, the cellular analog of sensitization, facilitation of nondecremented synaptic potentials, also emerges in the same late developmental stage (Nolen and Carew, 1988). In the present study, we have examined the development of sensitization in a different response system in Aplysia, escape locomotion.

We examined the emergence of sensitization in the escape locomotion system across a broad range of juvenile development: stages 10,11 , early 12 , late 12 , and early adult (stage 13 ). 
TEMPORAL EMERGENCE OF LEARNING

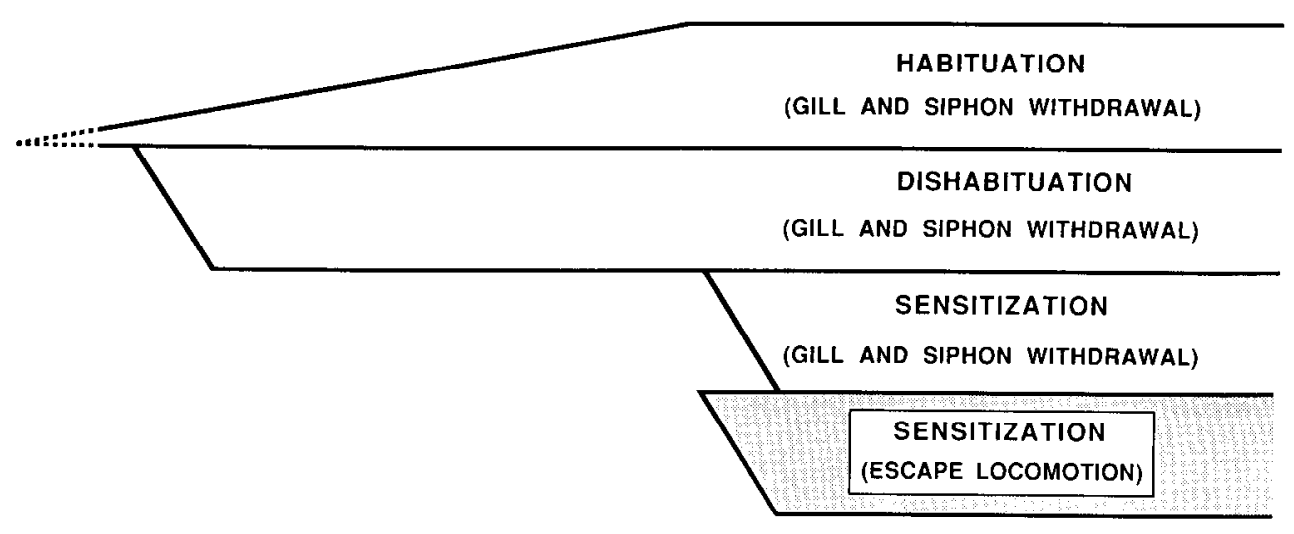

Figure 9. Schematic illustration of the developmental emergence of nonassociative learning in the gill and siphon withdrawal reflex and in the escape locomotion system (see text for discussion).

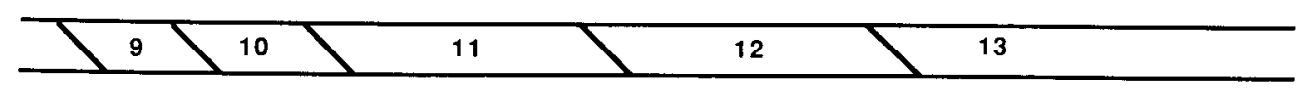

DEVELOPMENTAL STAGES

We found that sensitization was completely absent in early developmental stages; it did not appear until late stage 12 . This late stage is particularly interesting because, as mentioned above, it is during this stage that sensitization first appears in the gill and siphon withdrawal reflex, and the cellular analogs of sensitization are first detectable in the abdominal ganglion (Nolen and Carew, 1988; Rankin and Carew, 1988).

The timetables for the development of different forms of nonassociative learning in the gill withdrawal reflex and the escape locomotion system are shown in Figure 9. The emergence of sensitization in both the escape locomotion system and in the gill and siphon withdrawal reflex in the same late stage of juvenile development suggests the possibility that sensitization emerges as a single, unified process during development, and thus may be expressed in a variety of response systems simultaneously.

The fact that sensitization emerges within the same developmental stage in the escape locomotion system and the gill withdrawal reflex is interesting for 3 reasons. First, the 2 response systems have different birthdates. Escape locomotion emerges as early as stage 8 (unpublished observations), whereas siphon and gill withdrawal emerge several days later in stages 9 and 10, respectively (Kriegstein, 1977b; Rankin et al., 1987). Second, the gill and siphon withdrawal reflex and escape locomotion represent very different classes of response systems: $\mathrm{Si}$ phon and gill withdrawal are graded responses involving a restricted effector organ system (the mantle organs: gill, siphon, and mantle shelf), whereas escape locomotion is a cyclically recurring fixed action pattern, involving a widespread effector organ system (virtually the entire body, including the head and neck, the whole foot, the bilateral body walls, and the 2 parapodia). Third, the 2 response systems have very different underlying neural circuits: The gill and siphon withdrawal reflex is mediated by a relatively simple circuit located in the abdominal ganglion (Kupfermann and Kandel, 1969; Kupfermann et al., 1974; Byrne et al., 1978; Hawkins et al., 1981), whereas escape locomotion is mediated by more complex circuitry involving the coordination of triggering, oscillatory and effector circuits in the cerebral, pleural, and pedal ganglia (Jahan-Parwar and Fredman, 1978a, b; Hening et al., 1979). Thus, it is striking that, despite these several differences in ontogeny, response topography, and underlying circuit complexity, both response systems begin to express sensitization within the same developmental stage.

The emergence of sensitization in different response systems at the same time in development could imply a general developmental trigger for the expression of this form of learning. A recent study of the development of the central nervous system of Aplysia by Cash and Carew (1987) has shown that there is a highly nonlinear and dramatic proliferation of neurons in all ganglia, throughout the entire CNS in late stage 12 of juvenile development. Such a rapid increase in cell number at a specific developmental stage also implies the possibility of a specific developmental trigger for neuronal proliferation. Since the proliferation of central neurons occurs at the same stage of development (late stage 12) as the emergence of sensitization in at least 2 different response systems, this raises the interesting possibility that some common aspect of the developmental trigger for cell proliferation (e.g., either a general hormonal factor or the emergence of a particular class of facilitatory circuitry) could also participate in the expression of sensitization.

Although the coincident emergence of sensitization in both the gill withdrawal reflex and the escape locomotion system is consistent with the hypothesis that sensitization may emerge as a unified process, clearly more work is needed. To strengthen this hypothesis further, 2 kinds of studies will be necessary. First, it will be important to examine with greater precision the degree of synchrony in the emergence of sensitization in the 2 response systems. In the present study, we have localized the emergence of sensitization in both systems to a single developmental substage. It will now be important to refine the time scale to the specific day (or days) of emergence of sensitization in each system. Second, several other response systems in Aplysia exhibit sensitization. These include the tail withdrawal reflex (Walters et al., 1983b), the head withdrawal reflex (Walters et al., 1981), the inking response (Carew and Kandel, 1977), and feeding 
behavior (Kupfermann and Pinsker, 1968). Since these behaviors emerge at different times during development (Kriegstein et al., 1974; and unpublished observations), it will be extremely interesting to determine the timetable for the emergence of sensitization in each of these systems. Finally, it will be important to examine the emergence of sensitization in more than one response system within a single animal. Such a comparative analysis of the development of sensitization in a number of diverse response systems could provide further insights into the question of whether sensitization emerges as a unitary process during development.

\section{Development of escape locomotion}

After metamorphosis, juvenile Aplysia begin benthic locomotion by gliding on cilia that are located on the foot. This form of locomotion is gradually supplanted by a monotaxic pedal wave as the animal matures (Kriegstein et al., 1974), beginning during stage 11, and being complete only in stage 13 (adult) (Stopfer et al., 1987). Interestingly, we have observed that, although young juvenile animals (for example, stage 10) exclusively use ciliary gliding as their usual means of locomotion, they are, in fact capable of exhibiting more mature means of movement, such as stepping by means of pedal waves, which can be triggered by a noxious stimulus to the tail (Stopfer et al., 1987). Thus, the oscillatory neural circuitry required for pedal wave locomotion is in place and functional long (at least several weeks) before it is actually used as a normal means of locomotion by the animal.

Since juvenile animals exhibit 2 very different forms of escape locomotion (gliding and stepping) at different developmental stages, the possibility existed that the emergence of sensitization we observed in escape locomotion did not reflect the emergence of the learning process itself, but rather simply reflected the maturation of the response system. Specifically, it was possible that ciliary gliding could not be sensitized, but that pedal wave locomotion could be, and what appeared to be the emergence of the process of sensitization was actually the emergence of pedal wave locomotion, which exhibited sensitization as soon as this form of locomotion was developmentally expressed. However, this possibility could be ruled out since sensitization was still absent in developmental stages (such as stage 11 and early stage 12 ) when the animals could use pedal wave stepping as their means of locomotion. Furthermore, while the development of sensitization occurs quite abruptly during late stage 12 , the maturation of locomotor patterns proceeds gradually throughout the full range of juvenile stages studied (Stopfer et al., 1987). Thus, the emergence of sensitization in the escape locomotion system appears to reflect either the development of some aspect of the learning process itself or some means of initiating or triggering that process.

\section{Toward a cellular analysis}

In order to pursue the comparative analysis of the development of sensitization to a cellular level, it will be necessary to examine this question in behavioral systems in which the mechanisms of sensitization are relatively well understood. Important aspects of the neural circuitry underlying locomotion in Aplysia are already understood. Aplysia will initiate escape locomotion in response to stimuli delivered to virtually any site on the body, including the anterior tentacles, parapodia, siphon, tail, and the foot itself, demonstrating the presence of multiple triggering input pathways to the locomotor system. The foot is innervated primarily by 3 pedal nerves terminating in the anterior, middle, and posterior portions of the foot. Bilateral coordination during locomotion is controlled by the output of these nerves from central neuronal elements (Jahan-Parwar and Fredman, 1978a, b). Furthermore, it is known that locomotion in Aplysia is driven by a central program located in the circumesophageal ganglia; the basic oscillatory pattern underlying locomotion can be expressed by neural circuits in these ganglia in the absence of any feedback from the periphery (Hening et al., 1979). Several classes of neurons in the neural circuit for locomotion have been identified. These include 3 regional motor neuron populations and 4 unique, bilaterally symmetric motor neurons located in the pedal ganglia (Jahan-Parwar and Fredman, 1978b; Hening et al., 1979). Finally, the central program for locomotion can be triggered by the biogenic amine 5-HT and can be reversibly suppressed by extracts from the peptidergic bag cells that are responsible for coordinating a variety of egg-laying behaviors (Mackey and Carew, 1983).

Although important features of the neural mechanisms underlying escape locomotion are understood, the mechanisms underlying sensitization in this response system are not understood. In contrast, the mechanisms underlying sensitization in 2 other response systems in Aplysia have been extremely well analyzed. These are the gill and siphon withdrawal reflex (Klein and Kandel, 1978, 1980; Siegelbaum et al., 1982; for review, see Carew, 1987; Hawkins et al., 1987), and the tail withdrawal reflex (Walters et al., 1983a, b; Ocorr et al., 1985; for review, see Byrne, 1985). Since sensitization emerges at the same stage of development in the gill withdrawal reflex and the escape locomotion system, it will be of particular interest to examine the emergence of sensitization in the tail withdrawal reflex. Knowing its timetable will permit a comparative cellular analysis of the development of sensitization in 2 response systems that are well understood on cellular, biophysical, and molecular levels in the adult. Moreover, obtaining mechanistic insights into the development of sensitization in 2 well-understood reflex systems may, in turn, provide clues as to important elements and mechanisms to examine in the escape locomotion circuitry.

If sensitization emerges simultaneously in multiplc and independent response systems, this would imply the expression or induction of one or more critical integrating mechanisms that are responsible for an animal-wide activation of this form of learning. Such integrating mechanisms might include the induction of particular hormone, neurotransmitter, or facilitatory interneuronal systems, or the expression of one or more second messenger systems that are important for neuromodulation in the different response systems. These integrating mechanisms are likely to be important not only for the emergence of sensitization in different response systems during development, but also for the integration of sensitization in multiple response systems in the adult. Thus, by examining on behavioral and cellular levels the emergence of sensitization in multiple response systems during development in Aplysia, it may be possible to obtain important insights into the cellular and molecular mechanisms opcrating in the adult that are responsible for the coordinated expression of sensitization in multiple and diverse hehavioral systems.

\section{References}

Byrne, J. H. (1985) Neural and molecular mechanisms underlying information storage in Aplysia: Implications for learning and memory. Trends Neurosci. 8: 478-482. 
Byrne, J. H., V. F. Castellucci, and E. R. Kandel (1978) Contribution of individual mechanoreceptor neurons mediating defensive gill-withdrawal in Aplysia. J. Neurophysiol. 41: 418-431.

Carew, T. J. (1987) Cellular and molecular advances in the study of learning in Aplysia. In The Neural and Molecular Basis of Learning, J. P. Changeaux and M. Konishi, eds., pp. 177-204, Wiley, New York.

Carew, T. J., and E. R. Kandel (1977) Inking in Aplysia californica. I. The neural circuit of an all-or-none behavioral response. J. Neurophysiol. 40:692-707.

Carew, T. J., E. T. Walters, and E. R. Kandel (1981a) Classical conditioning in a simple withdrawal reflex in Aplysia. J. Neurosci. 1: 1426-1437.

Carew, T. J., E. T. Walters, and E. R. Kandel (1981b) Associative learning in Aplysia: Cellular correlates supporting a conditioned fear hypothesis. Science 211: 501-504.

Carew, T. J., R. D. Hawkins, and E. R. Kandel (1983) Differential classical conditioning of a defensive withdrawal reflex in Aplysia californica. Science 219: 397-400.

Cash, D., and T. J. Carew (1987) A quantitative analysis of the development of the CNS in juvenile Aplysia. Soc. Neurosci. Abstr. 13: 816.

Hawkins, R. D., V. F. Castellucci, and E. R. Kandel (1981) Interneurons involved in mediation and modulation of gill-withdrawal reflex in Aplysia. I. Identification and characterization. J. Neurophysiol.45: 304-314.

Hawkins, R. D., G. A. Clark, and E. R. Kandel (1985) Operant conditioning and differential classical conditioning of gill withdrawal in Aplysia. Soc. Neurosci. Abstr. 11: 796.

Hawkins, R. D., G. A. Clark, and E. R. Kandel (1987) Cell biological studies of learning in simple vertebrate and vertebrate systems. In Handbook of Physiology, Section I. The Nervous System, Vol. 6, F. Plum, ed., American Physiological Society, Bethesda, MD.

Hening, W. A., E. T. Walters, T. J. Carew, and E. R. Kandel (1979) Motorneuronal control of locomotion in Aplysia. Brain Res. 179:231253.

Jahan-Parwar, B., and S. M. Fredman (1978a) Control of pedal and parapodial movements in Aplysia. I. Proprioceptive and tactile reflexes. J. Neurophysiol. 41: 600-608.

Jahan-Parwar, B., and S. M. Fredman (1978b) Control of pedal and parapodial movements in Aplysia. II. Cerebral ganglion neurons. J. Neurophysiol. 41: 609-620.

Kandel, E. R. (1976) Cellular Basis of Behavior, Freeman, San Francisco.

Klein, M., and E. R. Kandel (1978) Presynaptic modulation of voltagedependent $\mathrm{Ca}^{++}$current: Mechanism for behavioral sensitization. Proc. Natl. Acad. Sci. USA 75: 3412-3516.

Klein, M., and E. R. Kandel (1980) Mechanism of calcium current modulation underlying presynaptic facilitation and behavioral sensitization in Aplysia. Proc. Natl. Acad. Sci. USA 77: 6912-6916.

Kriegstein, A. R. (1977a) Stages in the post-hatching development of Aplysia californica. J. Exp. Zool. 199: 275-288.

Kriegstein, A. R. (1977b) Development of the nervous system of Aplysia californica. Proc. Natl. Acad. Sci. USA 74: 375-378.

Kriegstein, A. R., V. F. Castellucci, and E. R. Kandel (1974) Metamorphosis of Aplysia californica in laboratory culture. Proc. Natl. Acad. Sci. USA 71: 3654-3658.

Kupfermann, I., and E. R. Kandel (1969) Neuronal controls of a behavioral response mediated by the abdominal ganglion of Aplysia. Science 164: 847-850.

Kupfermann, I., and H. Pinsker (1968) A behavioral modification of the feeding reflex in Aplysia californica. Commun. Behav. Biol. A 2: 13-17.

Kupfermann, I., T. J. Carew, and E. R. Kandel (1974) Local, reflex, and central commands controlling gill and siphon movements in Aplysia. J. Neurophysiol. 37: 996-1019.

Mackey, S., and T. J. Carew (1983) Triggering and modulation of escape locomotion in Aplysia by neurotransmitters and neuropeptides. J. Neurosci. 3: 1469-1477.
Mackintosh, N. J. (1974) The Psychology of Animal Learning, Academic, London.

Nolen, T. G., and T. J. Carew (1987) Analysis of non-decremented EPSPs prior to the emergence of sensitization reveals an inhibitory process in Aplysia. Soc. Neurosci. Abst. 13: 816.

Nolen, T. G., and T. J. Carew (1988) The cellular analog of sensitization in Aplysia emerges at the same time in development as behavioral sensitization. J. Neurosci. 8: 212-222.

Nolen, T. G., E. A. Marcus, and T. J. Carew (1987) Development of learning and memory in Aplysia. III. Central neuronal correlates. J. Neurosci. 7: 144-153

Ocorr, K. A., E. T. Walters, and J. H. Byrne (1985) Associative conditioning analog selectively increases cAMP levels of tail sensory ncurons in Aplysia. Proc. Natl. Acad. Sci. USA. 82: 2548-2552.

Peretz, B., and K. Lukowiak (1975) Age-dependent CNS control of the habituating gill withdrawal reflex and of correlated activity in identified neurons in Aplysia. J. Comp. Physiol. 103: 1-17.

Pinsker, H. M., I. Kupfermann, V. F. Castellucci, and E. R. Kandel (1970) Habituation and dishabituation of the gill-withdrawal reflex in Aplysia. Science 167: 1740-1742.

Pinsker, H. M., W. A. Hening, T. J. Carew, and E. R. Kandel (1973) Long-term sensitization of a defensive withdrawal reflex in Aplysia. Science 182: 1039-1042.

Rankin, C. H., and T. J. Carew (1986) Dishabituation and sensitization emerge as separate processes during development in Aplysia. Soc. Neurosci. Abstr. 12: 398.

Rankin, C. H., and T. J. Carew (1987a) Development of learning and memory in Aplysia. II. Habituation and dishabituation. J. Neurosci. 7: 133-143.

Rankin, C. H., and T. J. Carew (1987b) Analysis of the developmental emergence of sensitization in Aplysia reveals an inhibitory effect of a facilitatory stimulus. Soc. Neurosci. Abst. 13: 816 .

Rankin, C. H., and T. J. Carew (1988) Dishabituation and sensitization emerge as separate processes during development in Aplysia. J. Neurosci. 8: 197-211.

Rankin, C. H., M. Stopfer, E. A. Marcus, and T. J. Carew (1987) Development of learning and memory in Aplysia. I. Functional assembly of gill and siphon withdrawal. J. Neurosci. 7: 120-132.

Rayport, S. G., and J. S. Camardo (1984) Differential emergence of cellular mechanisms mediating habituation and sensitization in the developing Aplysia nervous system. J. Neurosci. 4: 2528-2532.

Siegel, S. (1956) Nonparametric Statistics for the Behavioral Sciences, McGraw-Hill, New York.

Sicgelbaum, S. A., J. S. Camardo, and E. R. Kandel (1982) Serotonin and cyclic AMP close single $\mathrm{K}^{\prime}$ channels in Aplysia sensory neurons. Nature 299: 413-417.

Stopfer, M., and T. J. Carew (1986) Development of escape locomotion in Aplysia. Soc. Neurosci. Abstr. 12: 399.

Stopfer, M., G. Schuerman, and T. J. Carew (1987) Developmental transition of locomotor programs in Aplysia californica. Soc. Neurosci. Abstr. 13: 1506.

Walters, E. T., T. J. Carew, and E. R. Kandel (1978) Conflict and response selection in the locomotor system of Aplysia. Soc. Neurosci. Abstr. 4: 209.

Walters, E. T., T. J. Carew, and E. R. Kandel (1979) Classical conditioning in Aplysia californica. Proc. Natl. Acad. Sci. USA 76:66756679.

Walters, E. T., T. J. Carew, and E. R. Kandel (1981) Associative learning in Aplysia: Evidence for conditioned fear in an invertebrate. Science 211: 504-506.

Walters, E. T., J. H. Byrne, T. J. Carew, and E. R. Kandel (1983a) Mechanoafferent neurons innervating the tail of Aplysia. I. Response properties and synaptic connections. J. Neurophysiol. 50: 1522-1542.

Walters, E. T., J. H. Byrne, T. J. Carew, and E. R. Kandel (1983b) Mechanoafferent neurons innervating the tail of Aplysia. II. Modulation by sensitizing stimuli. J. Neurophysiol. 50: 1543-1559. 